\title{
Chronic pancreatitis and mutations of the cystic fibrosis gene
}

Sharer N, Schwarz M, Malone G, et al. Mutations of the cystic fibrosis gene in patients with chronic pancreatitis. N Engl f Med 1998;339:645-52.

\begin{abstract}
Background: The pancreatic lesions of cystic fibrosis develop in utero and closely resemble those of chronic pancreatitis. Therefore, we hypothesized that mutations of the cystic fibrosis transmembrane conductance regulator (CFTR) gene may be more common than expected among patients with chronic pancreatitis. Methods: We studied 134 consecutive patients with chronic pancreatitis (alcohol-related disease in 71, hyperparathyroidism in 2 , hypertriglyceridemia in 1 , and idiopathic disease in 60). We examined DNA for 22 mutations of the $C F T R$ gene that together account for 95 percent of all mutations in patients with cystic fibrosis in the northwest of England. We also determined the length of the noncoding sequence of thymidines in intron 8 , since the shorter the sequence, the lower the proportion of normal CFTR messenger RNA. Results: The 94 male and $\mathbf{4 0}$ female patients ranged in age from $\mathbf{1 6}$ to 86 years. None had a mutation on both copies of the $C F T R$ gene. Eighteen patients (13.4 percent), including 12 without alcoholism, had a CFTR mutation on one chromosome, as compared with a frequency of 5.3 percent among 600 local unrelated partners of persons with a family history of cystic fibrosis $(P<0.001)$. A total of 10.4 percent of the patients had the $5 T$ allele in intron 8 (14 of 134), which is twice the expected frequency $(\mathbf{P}=0.008)$. Four patients were heterozygous for both a $C F T R$ mutation and the $5 T$ allele. Patients with a CFTR mutation were younger than those with no mutations $(P=0.03)$. None had the combination of sinopulmonary disease, high sweat electrolyte concentrations, and low nasal potentialdifference values that are diagnostic of cystic fibrosis. Conclusions: Mutations of the CFTR gene and the $5 T$ genotype are associated with chronic pancreatitis.
\end{abstract}

Cohn JA, Friedman KJ, Noone PG, et al. Relation between mutations of the cystic fibrosis gene and idiopathic pancreatitis. $N$ Engl f Med 1998;339:653-8.

Abstract

Background: It is unknown whether genetic factors predispose patients to idiopathic pancreatitis. In patients with cystic fibrosis, mutations of the cystic fibrosis transmembrane conductance regulator (CFTR) gene typically cause pulmonary and pancreatic insufficiency while rarely causing pancreatitis. We examined whether idiopathic pancreatitis is associated with $C F T R$ mutations in persons who do not have lung disease of cystic fibrosis. Methods: We studied 27 patients (mean age at diagnosis, 36 years), 22 of whom were female, who had been referred for an evaluation of idiopathic pancreatitis. DNA was tested for 17 CFTR mutations and for the $5 T$ allele in intron 8 of the CFTR gene. The $5 T$ allele reduces the level of functional CFTR and is associated with an inherited form of infertility in males. Patients with two abnormal CFTR alleles were further evaluated for unrecognized cystic fibrosis-related lung disease, and both base-line and CFTR-mediated ion transport were measured in the nasal mucosa. Results: Ten patients with idiopathic chronic pancreatitis (37 percent) had at least one abnormal $C F T R$ allele. Eight $C F T R$ mutations were detected (prevalence ratio, 11:1; 95 percent confidence interval, 5 to $23 ; \mathbf{P}<0.001)$. In three patients both alleles were affected (prevalence ratio, 80:1; 95 percent confidence interval, 17 to 379 ; $P<0.001)$. These three patients did not have lung disease typical of cystic fibrosis on the basis of sweat testing, spirometry, or base-line nasal potentialdifference measurements. Nonetheless, each had abnormal nasal cyclic AMP-mediated chloride transport. Conclusion: In a group of patients referred for evaluation of idiopathic pancreatitis, there was a strong association between mutations in the $C F T R$ gene and pancreatitis. The abnormal CFTR genotypes in these patients with pancreatitis resemble those associated with male infertility.

\section{Comment}

The common perception of cystic fibrosis (CF) is that of a recessive disorder presenting in infancy or early childhood with malabsorption and progressive lung disease. However, mild phenotypes have always been recognised. These individuals, who are pancreatic sufficient, present later, have less severe lung disease and significantly lower sweat $\mathrm{Cl}^{-}$ concentrations. Following identification of the CF gene in 1989 , it was realised that patients with a classic phenotype had severe mutations on both chromosomes, typically $\Delta \mathrm{F} 508$ which is present in $70 \%$ of $\mathrm{CF}$ chromosomes, whereas mildly affected individuals had one or more mild alleles. To date, more than 700 mutations have been identified in the CF gene; most, with the exception of G551D, G542X and $621+1$ (G-A) are rare, affecting $<1 \%$ of $\mathrm{CF}$ chromosomes.

The CF gene codes for a cyclic adenosine monophosphate (cAMP) mediated $\mathrm{Cl}^{-}$channel which has been identified in several epithelia including lung, biliary tract, pancreas, and vas deferens. ${ }^{1}$ This wide distribution accounts for the multi-organ involvement seen in cystic fibrosis. Channel function is mutation specific with five basic classes of mutation recognised (fig 1). ${ }^{2}$ Mutations that produce no CFTR protein (class I), where CFTR protein fails 

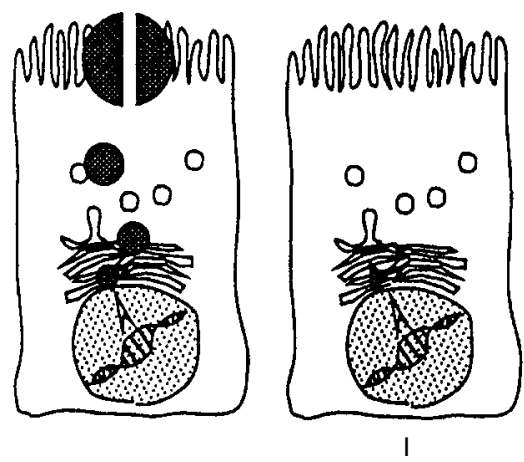

Normal

\author{
Nonsense \\ G542X \\ Frameshift \\ 394delTT \\ Splice junction \\ $1717-1 \mathrm{G} \rightarrow A$
}

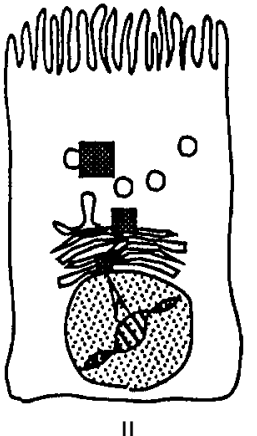

Missense

AA deletion

$\Delta \mathrm{F} 508$

(b)

(b)

(c)

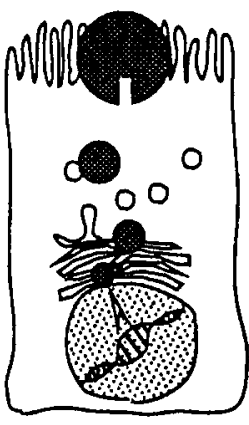

III

Missense G551D

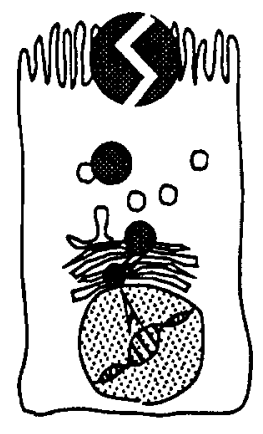

IV

Missense
R117H

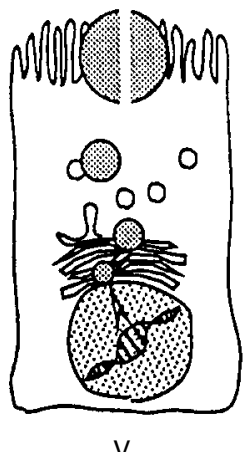

V

Missense
A455E

Alternative splicing

$3849+10 \mathrm{kbC} \rightarrow T$

(a)

(d)

(e)

(f)

Figure 1 Outcome of CFTR mutations compared with normally functioning (a) Cl- channels. (b) Class I: no CFTR protein formed. (c) Class II: CFTR mRNA formed but little functioning CFTR reaches the apical membrane. (d) Class III: CFTR fails to respond to cAMP. (e,f) Classes IV and V: reduced channel function or reduced systhesis of channel with normal properties. Reproduced with permission from Wilschanski et al. ${ }^{2}$

to reach the apical membrane because of defective processing (class II), or production of protein that fails to respond to cAMP (class III), have $<1 \%$ channel function and display a severe phenotype with pancreatic insufficiency. Mutations that produce a cAMP responsive channel with reduced conductance (class IV) or mutations that cause reduced synthesis or partially defective processing of normal CFTR (class V) cause mild disease, pancreatic sufficiency and often lower sweat electrolyte concentrations.

Recently, Schwartz's group in Manchester and Cohn et al from Duke University Medical Center, Durham, have described associations between mutant CFTR alleles ${ }^{1}$ and chronic pancreatitis. In the former study 18 (13.4\%) of 134 patients had CFTR mutations on one chromosome and in the latter $10(37 \%)$ of 27 patients with idiopathic chronic pancreatitis had at least one abnormal CFTR allele. This compares with an expected gene frequency of $5.3 \%$. In four and three patients, respectively, both alleles were affected; however, none had lung disease suggestive of cystic fibrosis nor typical sweat electrolyte abnormalities. Both studies identified patients with the 5T allele, a mutation in the non-coding sequence of thymidine residues on intron 8 which leads to a reduction in mRNA and low levels of CFTR protein. Homozygotes or compound heterozygotes with this mutation often have congenital bilateral absence of the vas deferens (CBAVD) and infertility.

Can these patients be considered to have cystic fibrosis? It is clear that the clinical spectrum of disease is broader than previously thought. Traditional diagnostic criteria (typical gastrointestinal or pulmonary disease and an abnormal sweat test) are no longer valid. A recent consen- sus report concluded that the diagnosis of cystic fibrosis can be confirmed by the presence of one or more characteristic phenotypic features, including pancreatitis, laboratory evidence of a CFTR mediated abnormality of ion transport, or the identification of disease causing mutations of each CFTR gene. ${ }^{3}$ Both groups used nasal potential difference measurements in an attempt to demonstrate abnormal CFTR function. ${ }^{4}$ Patients with recognised mutant alleles had higher potential differences than controls $(-10.5$ to $-24 \mathrm{mV})$ but well short of diagnostic potentials seen in patients with a classic phenotype $(-50 \mathrm{mV})$.

At present Sharer and Cohn's patients do not meet the criteria for cystic fibrosis. They should be considered a subgroup with impaired CFTR function which is possibly organ specific. There seems little justification for genetic testing of all patients with idiopathic chronic pancreatitis. However, the possibility of co-existing anomalies such as CBAVD should be considered.

C J TAYLOR

Department of Paediatrics,

Sheffield Children's Hospital,

Western Bank, Sheffield S10 2TH, UK

1 Zeng W, Lee MG, Yan M, et al. Immuno and functional characterization of CFTR in submandibular and pancreatic acinar and duct cells. Am $\mathcal{F}$ Physiol 1997;273:C442-55.

2 Wilschanski M, Zielenski J, Markiewicz D, et al. Correlation of sweat chloride concentration with classes of the cystic fibrosis transmembrane conductance regulator gene mutations. F Pediatr 1995;127:705-10.

3 Rosenstein BJ, Cutting GR. The diagnosis of cystic fibrosis: a consensus statement. F Pediatr 1998;132:589-95.

4 Middleton PG, Geddes DM, Alton EWFW. Protocols for in vivo measurement of the ion transport defects in cystic fibrosis nasal epithelium. Eur Respir f 1994;7:2050-6. 